\title{
Archéopages
}

Archéopages

Archéologie et société

46 | 2018

Maisons

\section{Une interprétation fonctionnelle des habitats rubanés}

L'apport de l'étude des comblements de trous de poteau

A functional interpretation of a Linear Pottery Culture (LBK) settlement. The contribution of studying posthole fillings

Una interpretación funcional de los hábitats de la cultura de la cerámica de bandas. El aporte del análisis de los rellenos de los orificios de poste

\section{Laura Berrio, Vincent Blouet et Julian Wiethold}

\section{OpenEdition}

Journals

Édition électronique

URL : https://journals.openedition.org/archeopages/3638

DOI : 10.4000/archeopages.3638

ISSN : 2269-9872

Éditeur

INRAP - Institut national de recherches archéologiques préventives

Édition imprimée

Date de publication : 1 décembre 2018

Pagination : 6-15

ISSN : 1622-8545

Référence électronique

Laura Berrio, Vincent Blouet et Julian Wiethold, « Une interprétation fonctionnelle des habitats rubanés », Archéopages [En ligne], 46 | 2018, mis en ligne le 01 décembre 2020, consulté le 02 juin 2021. URL : http://journals.openedition.org/archeopages/3638; DOI : https://doi.org/10.4000/ archeopages.3638 


\section{Une interprétation \\ fonctionnelle des habitats}

rubanés

L'apport de l'étude des comblements

de trous de poteau

Laura Berrio Université Paris 1 Panthéon-Sorbonne, UMR 8215 "Trajectoires »/UMR 7041 "ArScAn »

Vincent Blouet Ministere de la Culture

Julian Wiethold Inrap, UMR 628s, “ArTehis,

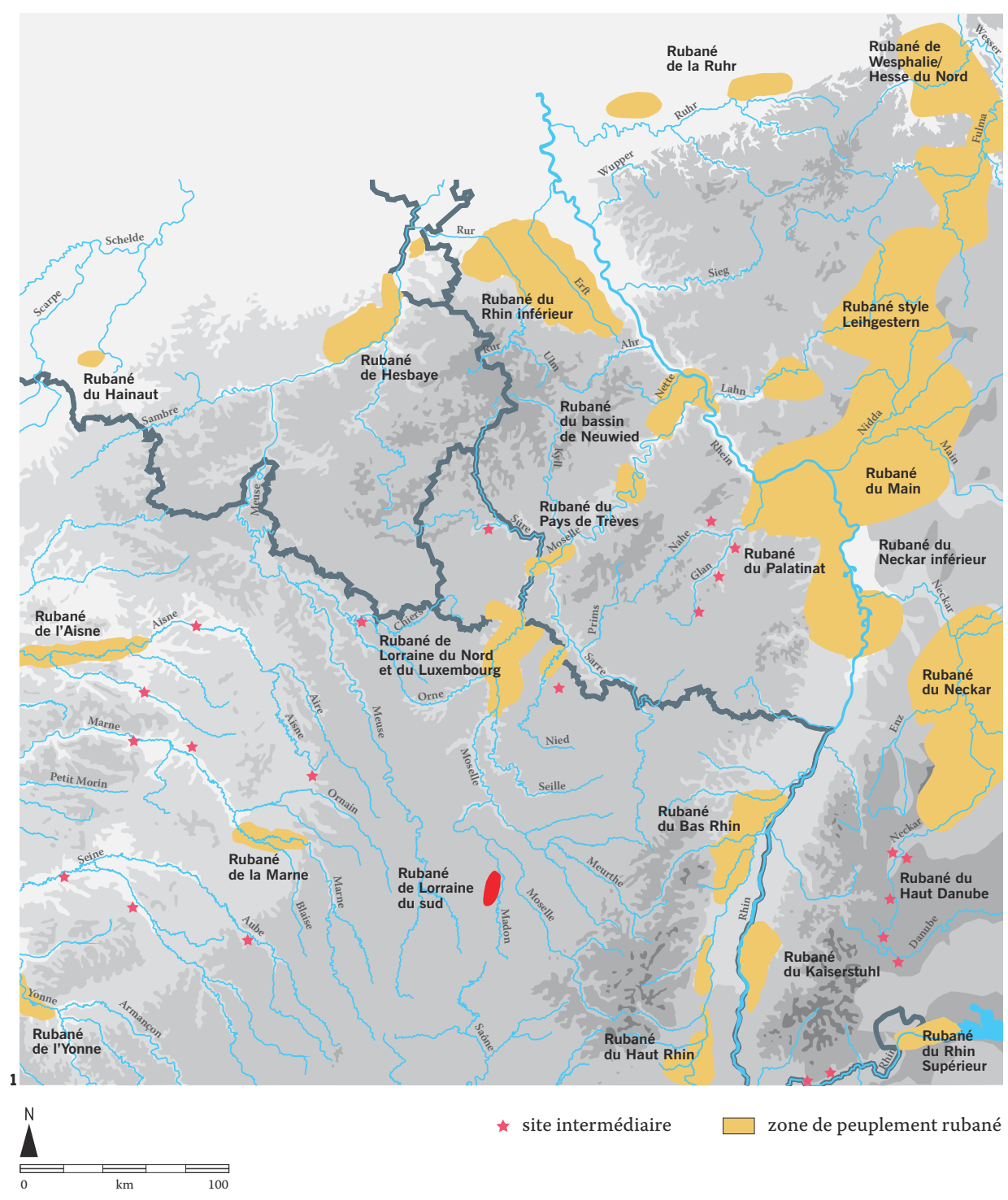

1.Zones de peuplement

rubané en Europe nord-

occidentale. En rouge : zone

d'implantation rubanée du

bassin supérieur de la Moselle

à laquelle appartient le site

de Marainville. 
Dans la majeure partie de l'Europe du Nord-Ouest, si on excepte les stations palafittiques, les témoins d'habitats protohistoriques se limitent à des structures excavées, les sols anciens étant rarement conservés sur les sites terrestres. Pour le Néolithique ancien rubané ${ }^{\mathbf{1}}$ (Blouet, 2006), les plans de construction sont restitués, lorsque l'érosion n'est pas trop importante, à partir des trous creusés pour implanter des poteaux et des traces laissées dans le substrat par le pourrissement des pièces de bois ayant constitué l'infrastructure. Pour reconstituer les élévations, l'archéologie expérimentale constitue un passage obligé, mais les données de terrain se résument souvent à la profondeur des poteaux supports de charpente ainsi qu'à leur rythme d'implantation. En l'absence des niveaux correspondant à la phase d'occupation, il est encore plus difficile de définir précisément la fonction d'un bâtiment comme des différentes parties qui le composent. Les analyses physico-chimiques sont parfois utilisées comme, par exemple, pour la mise en évidence de zones de stabulation par dosage de phosphate, mais l'emploi de ces méthodes nécessite des conditions de prélèvement et de conservation qui sont rarement réunies. Pour les interprétations fonctionnelles, à la suite notamment des travaux de Pierre Pétrequin (Pétrequin, 1984) et Anick Coudart (Coudart, 1998), il est donc largement fait appel aux comparaisons ethnographiques.

L'analyse du remplissage des trous de poteau est une méthode plus facile à mettre en œuvre, y compris dans le cadre de l'archéologie préventive, mais ce type d'études est encore rarement employé. Cette situation résulte des interrogations exprimées sur la correspondance entre la répartition des restes piégés dans les fantômes de poteau et celle des activités exercées dans les bâtiments, alors même que l'archéologie expérimentale et divers exemples de fouille permettent de lever en grande partie ces réserves. Pour ce qui concerne les exemples archéologiques, on peut ainsi rappeler que sur les sites rubanés de Sittard, Stein et Esloo (Pays-Bas), comme sur celui de Hienheim (Allemagne), les négatifs de poteau dessinant la trace de troncs refendus ont été interprétés comme résultant du vide laissé par le pourrissement du bois (Bakels, 1978). Pour ce qui concerne l'archéologie expérimentale, la construction puis le démontage d'une maison de l'âge du Fer à Pimperne (Grande-Bretagne) indiquent qu'après quatorze années d'utilisation, les objets trouvés dans les trous de poteau proviennent en majeure partie de la phase d'occupation (Reynolds, 1995). En effet, lors de la démolition du bâtiment, il a été observé que les bases de poteau étaient complètement décomposées, ce qui avait provoqué un déplacement des pierres de calage, les vides ayant été comblés par du sédiment et divers objets, produits du balayage du sol d'occupation. Les observations faites à Asparn an der Zaya
(Autriche) postérieurement à la démolition d'une maison expérimentale rubanée donnent des résultats comparables (Lenneis, Trebsche, 2013). Cinquante ans après la construction, la base des supports de charpente avait totalement été détruite par l'humidité, cette décomposition ayant permis l'infiltration des sédiments et du mobilier situé aux abords des poteaux.

On peut ainsi raisonnablement considérer que les vestiges piégés dans ces fantômes reflètent les activités pratiquées aux abords de chaque poteau. L'analyse pondérée des macrorestes végétaux (carporestes, charbons de bois) et des microvestiges (nodules de terre brûlée, esquilles) piégés après arrachement ou pourrissement des supports de charpente constitue donc un bon moyen pour appréhender la fonction des différentes parties d'un bâtiment.

Pour aborder ces problématiques, les archéologues intervenant sur les sites protohistoriques de Lorraine ont établi depuis une trentaine d'années un protocole de prélèvement systématique du remplissage du négatif des poteaux qui est mis en œuvre lorsque des ensembles cohérents sont mis au jour. Pour les âges du Bronze et du Fer, des résultats probants ont été obtenus concernant les emplacements de stockage des céréales à partir de la répartition des carporestes (De Hingh, 200o). Pour le Néolithique ancien, les études s'étaient jusqu'à présent concentrées sur la localisation des fours domestiques (Petitdidier et al., 2006 ; Blouet et al., 2007). L'analyse exhaustive des structures prélevées entre 1986 et 1988 sur le site de Marainville-sur-Madon (Vosges) permet d'illustrer les potentialités de cette méthode.

\section{Marainville, un habitat du Rubané récent et final en Lorraine}

La commune de Marainville-sur-Madon est située dans la plaine sous-vosgienne, à $10 \mathrm{~km}$ au nord-ouest de Charmes. À égale distance des zones d'implantations rubanées [ill. 1] de basse et haute-Alsace, de la moyenne Moselle et du bassin de la Marne, le site occupe une position particulièrement intéressante pour l'étude des interactions entre les différents groupes de la Linearbandkeramik (Blouet et al., 2013). Les styles céramiques comme les matériaux utilisés pour la confection de l'industrie lithique attestent de nombreux échanges avec l'Alsace et la Champagne, les contacts étant nettement plus distendus avec le Rubané de Lorraine du nord dont les plus proches habitats sont situés à moins de $80 \mathrm{~km}$. Les installations néolithiques sont implantées sur une petite croupe non inondable qui descend en pente douce jusqu'au Madon, affluent principal de rive gauche de la Moselle qui s'écoule à environ $100 \mathrm{~m}$ à l'est. Comme la plupart des habitats rubanés de Lorraine, le site de Marainville-sur-Madon n'est pas implanté sur des terrains lœssiques mais sur des limons colluvionnés, la bonne conservation
1. Le Néolithique ancien en Lorraine se déroule entre 5300 et 4.900 avant notre ère. 
2. Le site de Marainvillesur-Madon. L'occupation néolithique est constituée de cinq bâtiments

et d'une petite nécropole.

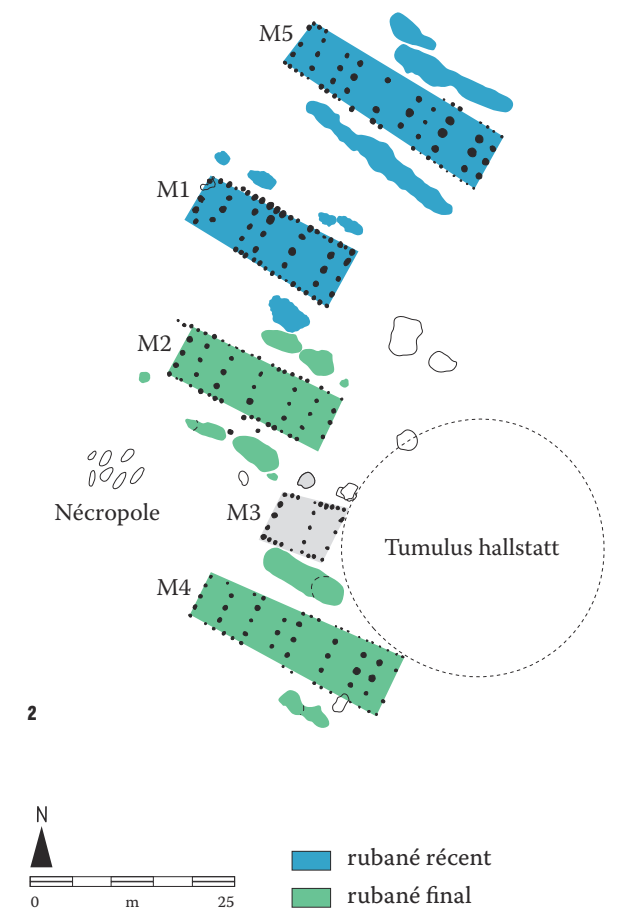

3. Emplacement des

prélèvements des

macrorestes végétaux

(carporestes, charbons de

bois) et des microvestiges

(nodules de terre brûlée,

esquilles). L'analyse a été

menée sur 135 prélèvements

provenant de 134 structures

(7 fosses latérales, 1 fosse

satellite, 89 trous de

poteau de tierce et 37 trous

de poteau de paroi)

Au total, 23060 carporestes

carbonisés (graines, fruits

et vannes de céréales) ont

été identifiés pour un total de

407,5 I de sédiment tamisé.

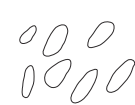

Nécropole

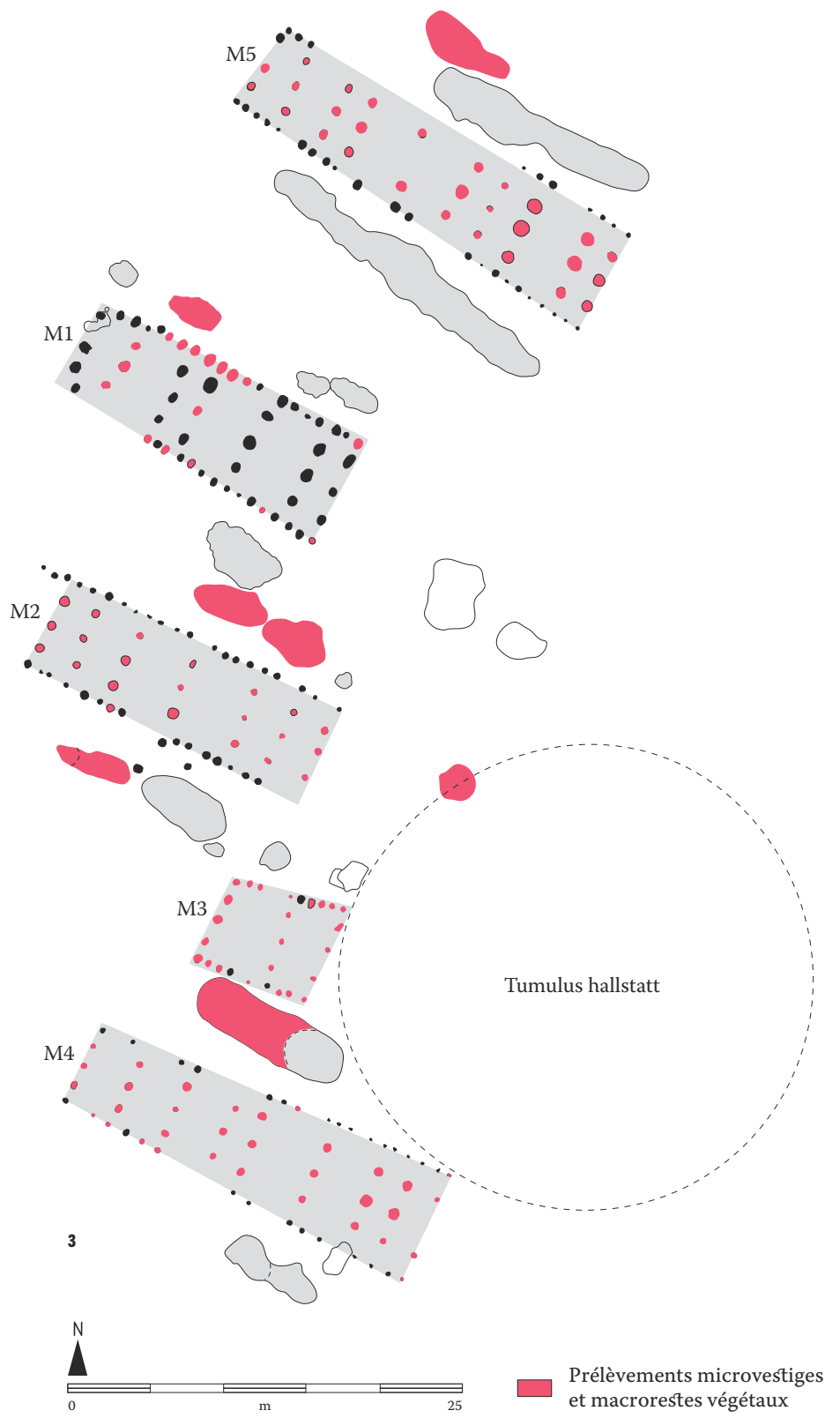

4. Les différentes parties fonctionnelles de la maison

M5. L'entrée du bâtiment se situe au sud-est.

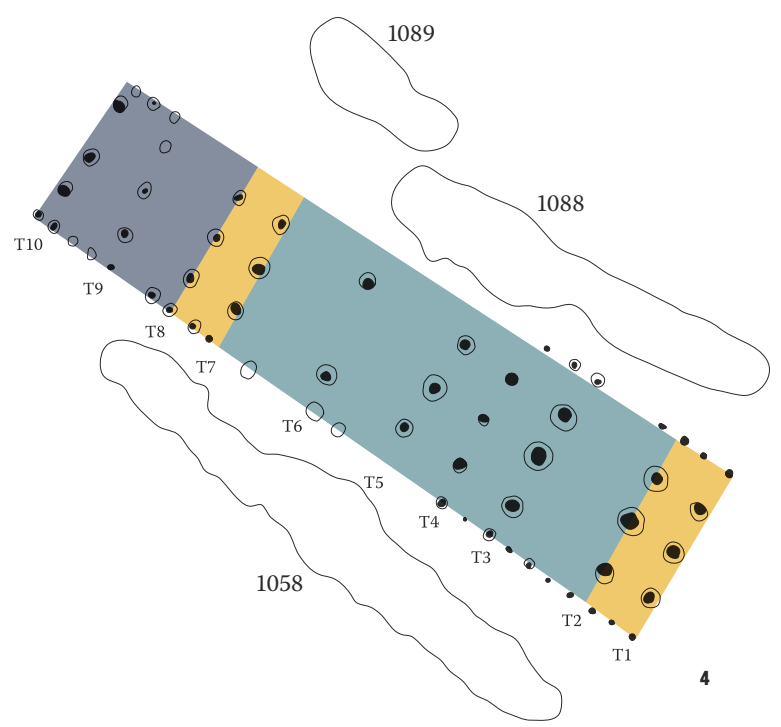


des trous de poteau et la profondeur des fosses témoignant d'une érosion peu importante.

Loccupation rubanée est constituée d'une petite nécropole et de cinq bâtiments orientés $\mathrm{SE}-\mathrm{NO}$ [ill. 2]. Quatre de ces constructions ( $\mathrm{M}_{5}, \mathrm{M}_{1}$, $\mathrm{M}_{2}$ et $\mathrm{M}_{4}$ ) présentent des fosses latérales à mobilier détritique qui montrent qu'il s'agit de maisons d'habitation. En revanche, l'interprétation de la cinquième $\left(\mathrm{M}_{3}\right)$, de petite dimension et de plan trapézoïdal irrégulier, est plus problématique : il pourrait s'agir de la partie arrière d'un bâtiment dont l'avant aurait été détruit par l'installation du tumulus hallstattien.

Létude céramique a permis de subdiviser l'occupation du site en deux phases. La première correspond à la fin du Rubané récent et associe les bâtiments M5 et M1, tandis que la seconde est attribuable au Rubané final et regroupe les bâtiments $\mathrm{M}_{2}$ et M4. Diverses observations (architecture et orientation des bâtiments, distance entre les constructions, différences dans le spectre des matériaux utilisés pour l'industrie lithique...) laissent penser que ces constructions ne sont pas synchrones. Sur ces fondements, l'hypothèse d'une seule unité d'habitation reconstruite à quatre, voire cinq reprises si le bâtiment $\mathrm{M}_{3}$ est une maison en partie érodée, est la plus vraisemblable, en conformité avec le modèle de Hofplatz proposé pour le plateau d'Aldenhoven (Lüning, 1998).

Des prélèvements de sédiment ont été réalisés dans la plupart des fosses qui recelaient des couches charbonneuses. À l'exception de la maison M1, dont la partie avant a été explorée en 1986, les poteaux de tierce ont tous été prélevés [ill. 3], tandis que ceux de paroi l'ont été de façon plus aléatoire eu égard à leur degré de conservation. Les tierces sont les poteaux qui structurent l'espace interne des bâtiments rubanés. Il s'agit d'une succession de poteaux alignés trois par trois perpendiculairement à la longueur de la maison, en rangées parallèles entre elles et délimitant des travées. Elles sont numérotées en partant de ce qui est considéré comme l'entrée des bâtiments, orientée généralement vers le sud-est dans toute l'aire rubanée. Les prélèvements correspondent soit au remplissage du négatif des poteaux, soit aux fosses de creusement lorsque ces derniers n'étaient pas visibles. L'ensemble des sédiments a été tamisé sur une colonne de $5 \mathrm{~mm}, 2 \mathrm{~mm}$ et $0,315 \mathrm{~mm}$, les macrorestes végétaux étant recueillis par flottation.

\section{L'étude architecturale de la maison $\mathrm{M}_{5}$}

La maison $\mathrm{M}_{5}$ est sans doute la première implantée sur le site de Marainville-sur-Madon, les fosses latérales associées ayant livré du mobilier attribuable à la fin du Rubané récent $\mathrm{A}$ (Blouet et al., 2013, vol. 1, p. 309). Sous le décapage, la profondeur conservée des tierces de ce bâtiment est au minimum de o,20 m (support faîtier du pignon arrière) et au maximum de o,65 $\mathrm{m}$ (poteaux des supports latéraux de pannes du corridor 2. It : Indice de trapézité. avant). La totalité des poteaux de paroi dont le négatif était observable sont des bois refendus, au contraire des tierces qui, pour les trois quarts, ne le sont pas. Mis à part quelques manques, la paroi sud est relativement bien conservée tandis que l'érosion semble avoir été plus forte sur la paroi nord. Bien que le pignon arrière soit situé à la limite de fouille, l’organisation générale des poteaux et l'interruption de la paroi sud au niveau de la première tierce permettent de penser que le plan de la construction est complet [ill. 4].

Si l'on fonde l'étude architecturale sur le plan de fouille et l'analyse métrique, il s'agit d'une maison de type 1 de Modderman (Großbau ou grande maison) légèrement trapézoïdale $\left(\mathrm{It}^{2}=0,02\right)$, d'une longueur de 25,50 $\mathrm{m}$ pour une largeur de 5,60 m à l'arrière et 6,70 $\mathrm{m}$ à l'avant dont l'orientation est de 63 gr ouest. Succédant au corridor avant (tierces T1 et $\mathrm{T}_{2}$ ), un groupement de neuf poteaux (tierces $\mathrm{T}_{3}$, $\mathrm{T}_{4}$ et $\mathrm{T}_{5}$ ), dont certains de fort calibre et profondément ancrés dans le sol, suggèrent, dans un premier temps, l'existence d'une partie frontale dotée d'un grenier surélevé (Coudart, 1998). Dans cette hypothèse, la partie centrale, d'une superficie de $48 \mathrm{~m}^{2}$, classiquement interprétée comme la pièce d'habitation, serait comprise entre ce grenier et le corridor arrière (tierces $\mathrm{T}_{7}$ et $\mathrm{T} 8$ ), c'est-à-dire entre les tierces $\mathrm{T}_{5}$ et $\mathrm{T}_{7}$. Toutefois, l'étude de la répartition des microvestiges et des macrorestes végétaux, complétée par celle de la profondeur des poteaux, montre que cette interprétation est erronée. En effet, la tierce $\mathrm{T}_{4}$ se distingue de celles qui l'encadrent $\left(\mathrm{T}_{3}\right.$ et $\left.\mathrm{T}_{5}\right)$ par des poteaux de plus petit gabarit et de faible enfoncement mais aussi par la quasi-absence de microvestiges et de macrorestes végétaux dans les refus de tamis. Même si elle s'inscrit dans le plan général du bâtiment, il est donc vraisemblable que cette tierce n'était pas fonctionnelle au même moment que celles qui l'entourent. Il pourrait ainsi s'agir d'une structure de type échafaudage, démonté immédiatement après l'édification du bâtiment, ce qui expliquerait l'absence de vestiges issus de la phase d'occupation. L'hypothèse la plus plausible est cependant que l'on a affaire à une reprise en sousœuvre des pannes intervenant peu de temps avant l'abandon du bâtiment, l'absence de microvestiges et de macrorestes végétaux s'expliquant alors par le caractère éphémère de la réfection.

Dans un cas comme dans l'autre, l'interprétation fonctionnelle du bâtiment doit être révisée.

L'hypothèse initiale d'un grenier surélevé ferait ainsi place à celle d'une partie centrale de $93 \mathrm{~m}^{2}$, comprise entre les corridors avant et arrière et composée de quatre travées (tierces $\mathrm{T}_{3}, \mathrm{~T}_{5}$ et T6).

\section{Une maison incendiée?}

En première analyse, l'abondance (plus de $29 \mathrm{~kg}$ ) des nodules de terre rubéfiée, ajoutée à celle des carporestes carbonisés (Berrio, 2012), pourrait laisser penser que la maison M5 a été détruite par le feu. Dans les habitats ouverts, en l'absence de sol conservé, les éléments pouvant correspondre à des 
concentration de nodules

de terre rubéfiée à l'avant

(sud-est) de la partie centrale

indique vraisemblablement

l'emplacement du four

domestique.

poteaux : poids torchis (g) fosses : poids torchis (g) par litre de sédiment

\begin{tabular}{|c|c|c|}
\hline $\mathrm{o}$ à < $10 \mathrm{~g}$ & $\square$ & 0-1 ooo g \\
\hline $10 \mathrm{~g}$ à < $50 \mathrm{~g}$ & & $1001-2000 \mathrm{~g}$ \\
\hline $50 \mathrm{~g}$ à $<100 \mathrm{~g}$ & & $2001-3000 \mathrm{~g}$ \\
\hline $\begin{array}{l}>=100 \mathrm{~g} \\
\text { poteaux paroi }\end{array}$ & & $4070 \mathrm{~g}$ \\
\hline
\end{tabular}
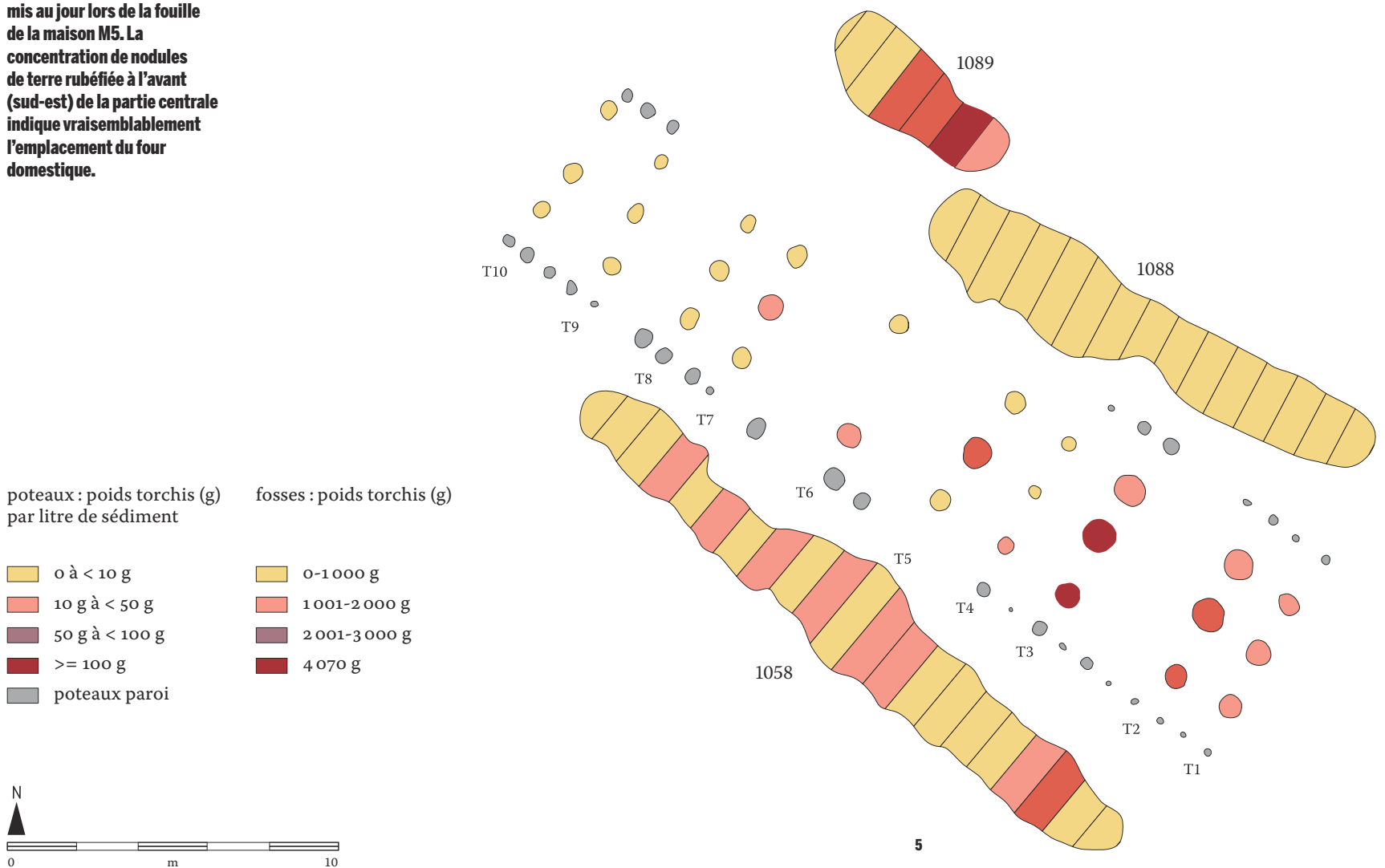

6. Répartition des densités de carporestes dans le bâtiment M5. Les plus faibles densités se trouvent dans la fosse 1089 ainsi que dans les tierces T1 à T4, des densités plus importantes sont présentes dans les tierces $\mathrm{T} 6$ à T9, et les densités

les plus élevées se trouvent dans les tierces $\mathrm{T} 5$ et $\mathrm{T} 9$.

poteaux et fosse :

carporestes par litre de sédiment

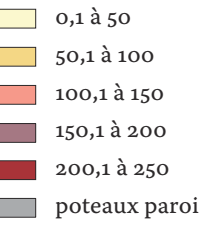

$\Lambda^{N}$

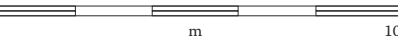

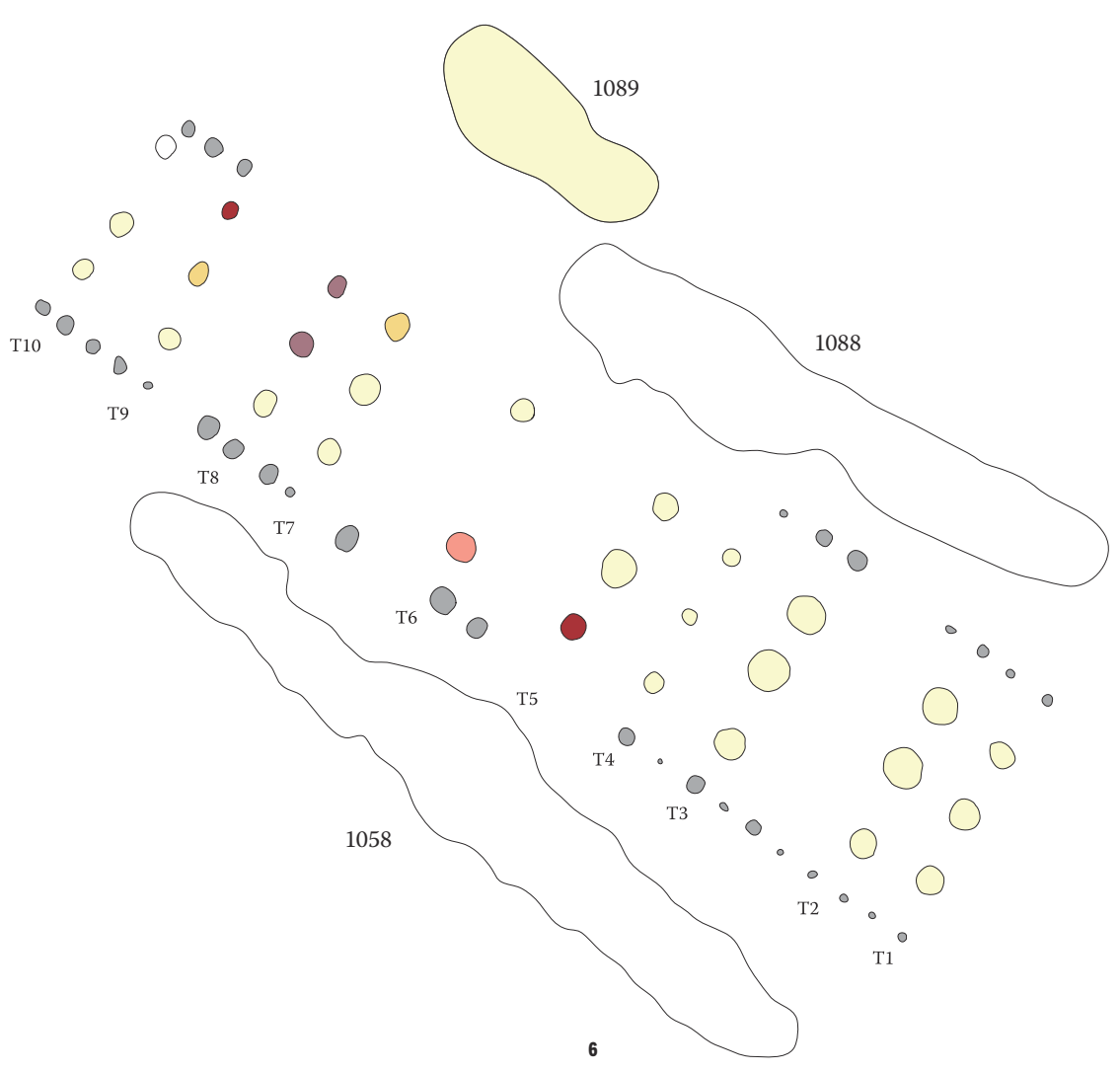


témoins d'incendie se limitent le plus souvent à des concentrations de charbon de bois au niveau des fantômes de poteau et à des fragments de torchis interprétés comme les vestiges de murs brûlés. Quatre exemples de bâtiments brûlés du Néolithique ancien en Écosse, connus comme « halles en bois » (timber halls) - Balbridie (Fairweather, Ralston, 1993), Lockerbie (Kirby, 2011), Claish (Barclay et al., 2002) et Warren Field (Murray et al., 2009) -, se caractérisent par la présence de torchis brûlé, d'assemblages carpologiques présentant un spectre varié en provenance des trous de poteau et de concentrations élevées de charbons de bois. Le brûlage expérimental de poteaux de bois (Murray et al., 2009) démontre que les poteaux ne sont pas touchés par le feu en dessous du niveau du sol. Pour les cas archéologiques, cela suggère que le bois a été carbonisé au-dessus du niveau du sol et que les charbons et autres restes sont tombés dans les trous de poteau lorsque la portion non brûlée des poteaux a pourri.

Dans le cas de la maison M5 de Marainville, on note d'abord la rareté des charbons de bois qui constituent un poids total de moins de $20 \mathrm{~g}$ récoltés pour l'ensemble des structures composant l'unité d'habitation. Les études de répartition [ill. 5] montrent quant à elles que la dispersion des nodules de terre brûlée ne correspond pas à celle qui résulterait d'un incendie. Dans les fosses latérales, les blocs de torchis ne sont pas répartis uniformément le long des parois mais sont, pour plus d'un tiers, concentrés dans une fosse (st. 1089) située à l'arrière nord du bâtiment. Ce type d'amas, qui témoigne du rejet intentionnel de ces matériaux encombrants à l'extérieur des espaces utiles, se retrouve sur plusieurs sites rubanés de la Grande Région (Sarre-Lorraine-Luxembourg).

En ce qui concerne la répartition des nodules brûlés dans les fantômes de poteau, on relève une absence totale dans la partie arrière de la maison, secteur où se retrouvent pourtant des accumulations de carporestes carbonisés. À l'inverse, dans la partie centrale, on constate des concentrations de terre cuite dans les tierces des deuxième et troisième travées. De telles concentrations de torchis dans les poteaux de la pièce résidentielle se retrouvent sur les trois autres maisons de Marainville-sur-Madon mais aussi en Lorraine du Nord, notamment sur les sites de Metz «Ban-de-Devant-lès-Ponts » et Montenach « Kirchgasse ». L'hypothèse la plus vraisemblable est que ces restes matérialisent l'emplacement d'une structure de combustion, de type four à coupole sur armature en clayonnage, les rejets retrouvés dans les fosses extérieures résultant quant à eux de phases de réfection de ces fours (Blouet, 2017). Ces observations sont à mettre en parallèle avec la découverte de quatre foyers dans une maison à Postoloprty en République tchèque (Soudský, 1969).

L'analyse de la répartition des charbons de bois et des terres cuites récoltés au tamisage amène à écarter l'hypothèse d'un incendie et permet de localiser une structure de combustion implantée vraisemblablement au niveau de la troisième tierce.

\section{L'apport des carporestes}

L'interprétation des données carpologiques issues des fosses latérales ne pose pas de problème particulier. Comme cela a déjà été observé sur plusieurs sites rubanés (Kreuz, 2012 ; Kreuz et al., 2005), ces déchets domestiques sont associés aux traitements des récoltes et à la préparation d'aliments. En revanche, l'interprétation du matériel issu des trous de poteau est un peu plus problématique vu l'abondance des carporestes (3540 NMI), exceptionnelle pour un site rubané et qui pose la question de l'origine des restes.

Les datations radiocarbones pratiquées sur les graines montrent que ces restent correspondent à l'occupation rubanée du site.

Il est probable que ces carporestes proviennent d'activités culinaires ayant provoqué leur carbonisation, leur piégeage dans les trous de poteau résultant des balayages du sol pendant l'occupation du bâtiment. Cependant, il n'est pas possible d'écarter l'hypothèse selon laquelle ces carporestes auraient trouvé leur place définitive après l'abandon du bâtiment, à la suite de la récupération des pièces de bois encore utilisables pour la construction d'une nouvelle maison ou comme bois de chauffage. Une telle destruction a pu être suivie d'un nettoyage du sol abandonné, éventuellement réalisé en utilisant le feu, ce qui aurait augmenté la probabilité de carbonisation et donc de conservation des carporestes issus des activités domestiques.

L'analyse spatiale des densités de carporestes [ill. 6] comme celle des céréales, des légumineuses et des plantes adventices [ill. 7 et 8] montrent une distribution hétérogène au sein de la maison $\mathrm{M}_{5}$ (Berrio, 2012). Le corridor avant se caractérise par la prédominance de caryopses de céréales vêtues, la présence d'un faible pourcentage de légumineuses et de vannes (bases de glumes et bases d'épillets des blés vêtus) - à l'exception d'un trou de poteau où les vannes sont majoritaires -, ainsi qu'un pourcentage très faible de plantes adventices. Dans la partie centrale, les caryopses de céréales vêtues prédominent, les vannes se trouvent en faible pourcentage alors que les légumineuses, les caryopses de céréales nues et les plantes adventices sont présents en très faible pourcentage. Le corridor arrière, la partie arrière et la fosse 1089 se distinguent quant à eux par la prédominance de légumineuses alors que les caryopses de céréales occupent une place importante. Le pourcentage de caryopses de céréales nues est légèrement plus élevé que celui de céréales vêtues dans la partie arrière. Les vannes sont présentes en faible pourcentage dans le corridor arrière et en très faible pourcentage dans la partie arrière alors que les plantes adventices sont quasi absentes dans les deux secteurs. 
7. Distribution des catégories de plantes dans le bâtiment M5. Les tierces T1 et T2 se caractérisent par une prédominance de caryopses de céréales vêtues, un faible pourcentage de vannes et de légumineuses, et un très faible pourcentage de plantes adventices ; les tierces T3, T5 et T6 par une prédominanc de caryopses de céréales vêtues, un faible pourcentage de vannes et un très faible pourcentage de plantes adventices; la fosse 1089 ainsi que les tierces 7 à $T 10$ par une prédominance de légumineuses, une place importante des céréales, des plantes adventices quasi absentes dans les trous de poteau et absentes dans la fosse, et une absence de vannes à l'exception de la tierce 77 où elles sont présentes en faible pourcentage.

N.B. Échantillons présentés $\mathrm{si} \geq 20$ carporestes

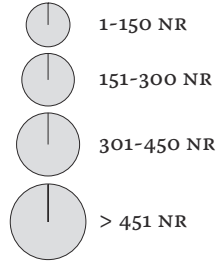

céréales, caryopses

céréales, vannes (bases de glume)

légumineuses

plantes adventices
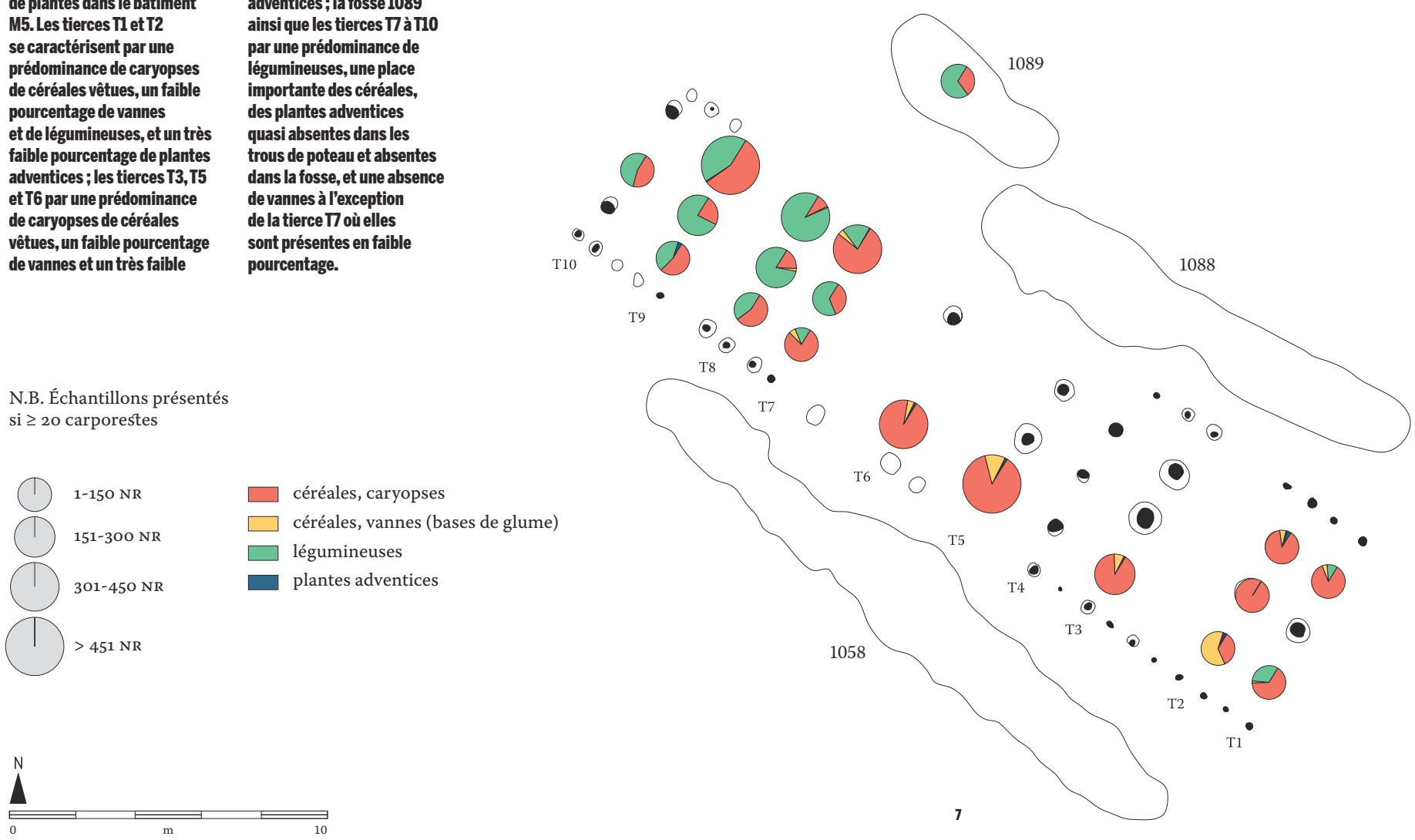

8. Distribution des céréales dans le bâtiment M5.

La fosse 1089 se caractérise par une prédominance de caryopses de céréales, les tierces $\mathrm{T1}$ à $\mathrm{T}$ par une prédominance de caryopses de céréales vêtues et un faible pourcentage de caryopses de céréales nues et de

N.B. Échantillons présenté si $\geq 20$ carporestes

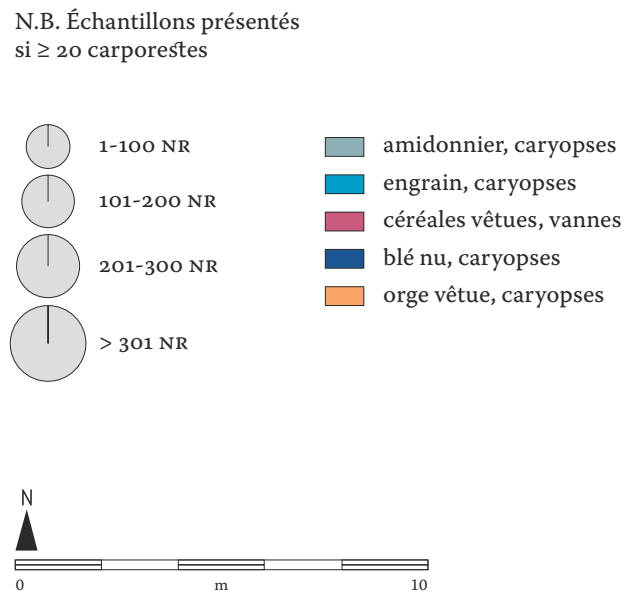

vannes, à l'exception d'un trou de poteau de la tierce T2 où les vannes prédominent La tierce $T 9$ se caractérise par la prédominance de caryopses de céréales nues, un faible pourcentage de caryopses de céréales vêtues et un très faible pourcentage de vannes.

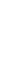




\section{Quel modèle d'organisation de l'espace domestique?}

À Langweiler 8 en Rhénanie (Allemagne), des différences importantes dans le spectre carpologique des maisons avec et sans partie frontale de type grenier ont été observées, les fosses latérales des maisons comportant une partie frontale ayant livré des proportions plus élevées de vannes et de plantes adventices que les autres maisons. Ceci a permis de conclure que le traitement et la gestion des récoltes étaient dévolus aux maisons comportant une partie frontale de type « grenier » (Knörzer, 1988). À Bylany en Bohême (République tchèque), une fosse a été mise au jour dans chacune des parties arrière des maisons 96, 306 et 679, ces données ont été interprétées comme une sorte de cave et la partie arrière elle-même comme un cellier (Pavlů, 200o).

À Marainville, la distribution hétérogène des restes reflète très probablement l'organisation interne de l'espace domestique. Une fois écarté le cas du bâtiment $\mathrm{M}_{5}$, il n'existe pas de maison à grenier surélevé. Au vu des faibles densités de carporestes provenant du corridor avant $\mathrm{du}$ bâtiment $M_{5}$, dont le spectre se caractérise par une prédominance de caryopses de céréales ainsi qu'un faible pourcentage de vannes, de légumineuses et de plantes adventices, la fonction de ce secteur de la maison reste difficile à interpréter. Cependant, les données suggèrent que, dans ce vestibule par lequel on accédait à la zone domestique, auraient probablement eu lieu certaines activités domestiques en relation avec le traitement des céréales, par exemple lorsque les conditions climatiques ne favorisaient pas le travail à l'extérieur. Il s'agirait également d'une aire de circulation et d'évacuation des déchets correspondant à l'entrée du bâtiment.

En ce qui concerne la partie centrale, elle semble avoir été le lieu d'activités domestiques qui se concentrent dans sa partie sud. Les concentrations de carporestes carbonisés, le pourcentage élevé de caryopses de céréales vêtues et de légumineuses ainsi que la présence de vannes et de quelques plantes adventices pourraient être interprétées comme une zone consacrée à la préparation d'aliments où, probablement, le traitement des céréales aurait eu lieu au fur et à mesure des besoins. À Vaihingen/Enz dans le Bade-Wurtemberg (Allemagne), les restes de plantes adventices dans les trous de poteau ont été interprétés comme une possible preuve du nettoyage des céréales à l'intérieur de la maison (Bogaard, 2011). Il est à noter que le site de Bylany a livré un pot de stockage enterré sous le plancher de la maison 96, placé dans le coin sud-est de la partie centrale et interprété comme un pot de stockage pour les céréales ou pour l'eau (Pavlů, 200o).

Le corridor arrière présente des densités élevées et le spectre carpologique partage des caractéristiques de la partie arrière (la prédominance de légumineuses) et de la partie centrale (la présence de vannes et le pourcentage important de caryopses de céréales vêtues).

Ces aspects sont semblables à ceux trouvés dans la fosse 1089 située en face, le long de la paroi nord, et suggèrent l'existence d'une aire de circulation entre la partie centrale et la partie arrière, possiblement avec une ouverture vers l'extérieur qui permettait l'évacuation des déchets du secteur nord du bâtiment.

Quant à la partie arrière orientée vers le nordouest, elle présente les densités les plus élevées caractérisées par la prédominance de légumineuses et de céréales nues. En revanche, les proportions de vannes et de céréales vêtues y sont plus faibles que dans la partie centrale. Étant donné que les céréales vêtues sont les principales denrées d'origine végétale au Rubané, leur faible proportion dans la partie arrière fait penser que la préparation d'aliments ne s'y déroulait pas. Ces concentrations de légumineuses et de céréales nues pourraient plutôt révéler une zone de stockage à court terme où seraient conservées les provisions de la maisonnée consommées assez rapidement. Le caractère rapide de l'évacuation du stock est important si nous prenons en compte deux aspects. D'une part, l'exposition nord-ouest de cette pièce rend cet endroit de la maison un peu contraignant pour un stockage à long terme. En effet, les changements brusques de température pourraient entraîner assez rapidement une dégradation des denrées alimentaires. D'autre part, les conditions de stockage des légumineuses et des céréales nues ne sont pas les mêmes que celles des céréales vêtues. En effet, les légumineuses sont généralement stockées en graines, la technique de stockage en gousse nécessitant des capacités de stockage importantes - le volume d'une gousse est jusquà 6,5 fois plus élevé que celui des graines. De plus, il est difficile de vérifier si les graines enfermées dans leurs gousses ont fait l'objet d'une attaque d'insectes. En ce qui concerne les céréales nues, la chaîne de traitement comporte une étape de dépiquage lors de laquelle les épis sont fragmentés et les caryopses libérés de la balle. Ensuite, le stockage se fait sous forme de caryopses nettoyés. Sous ces conditions de stockage, les graines et les caryopses sont peu protégés et particulièrement exposés à l'humidité ambiante ainsi qu'aux ravages causés par les insectes et les moisissures.

L'étude de répartition pondérée issue du tamisage des poteaux de la maison M5 de Marainville permet de préciser l'architecture et la fonction des différentes parties de cette habitation. À la différence de ce que suggéraient les premières conclusions (Blouet et al., 2013, vol. 2, p. 155), ce bâtiment n'est pas doté d'un grenier avant et, au contraire, les réserves en légumineuses et céréales nues semblent avoir été stockées dans la pièce située après le corridor arrière. Au niveau du corridor avant, l'ubiquité des des micro-vestiges 


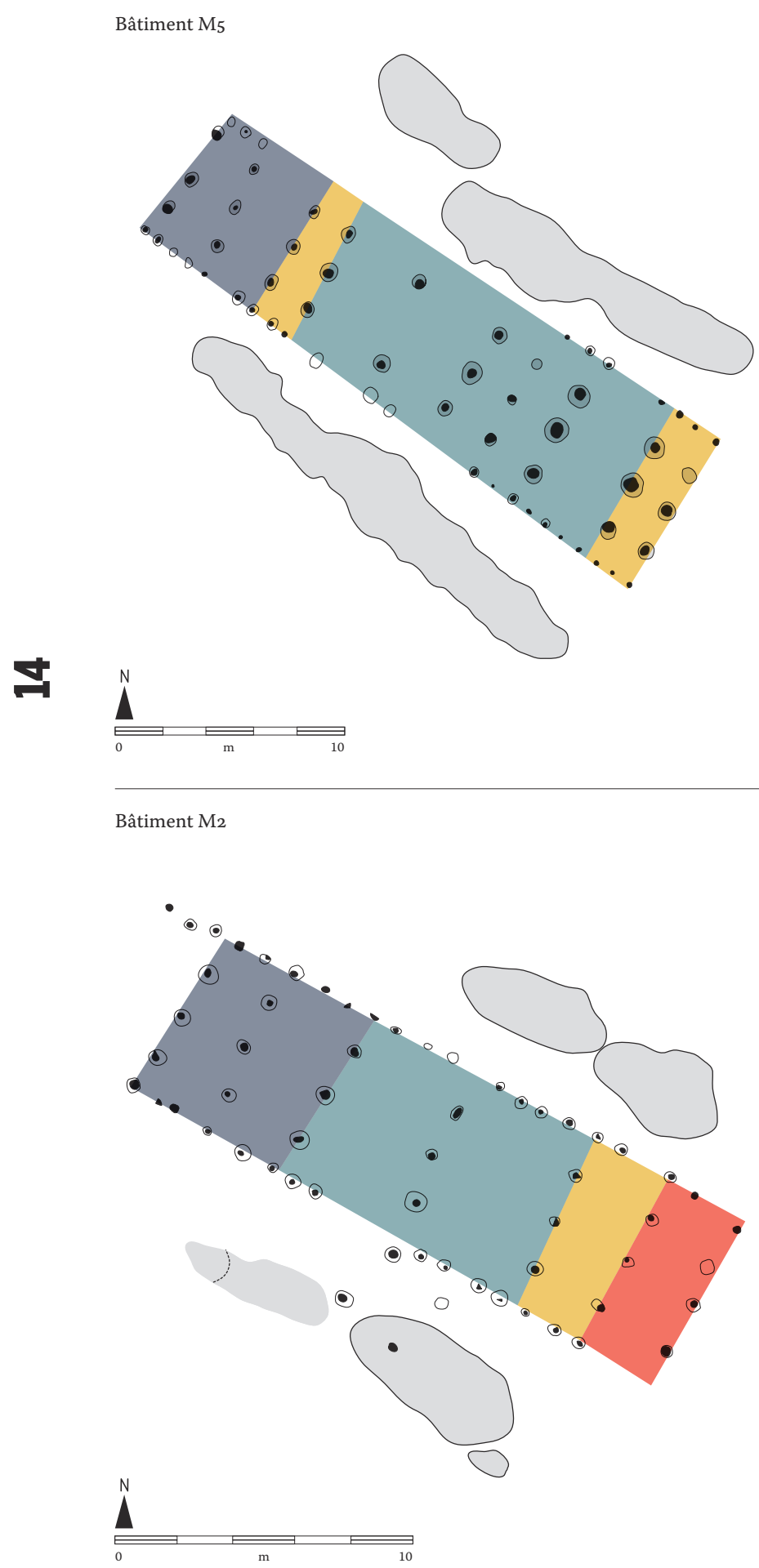

Bâtiment M1
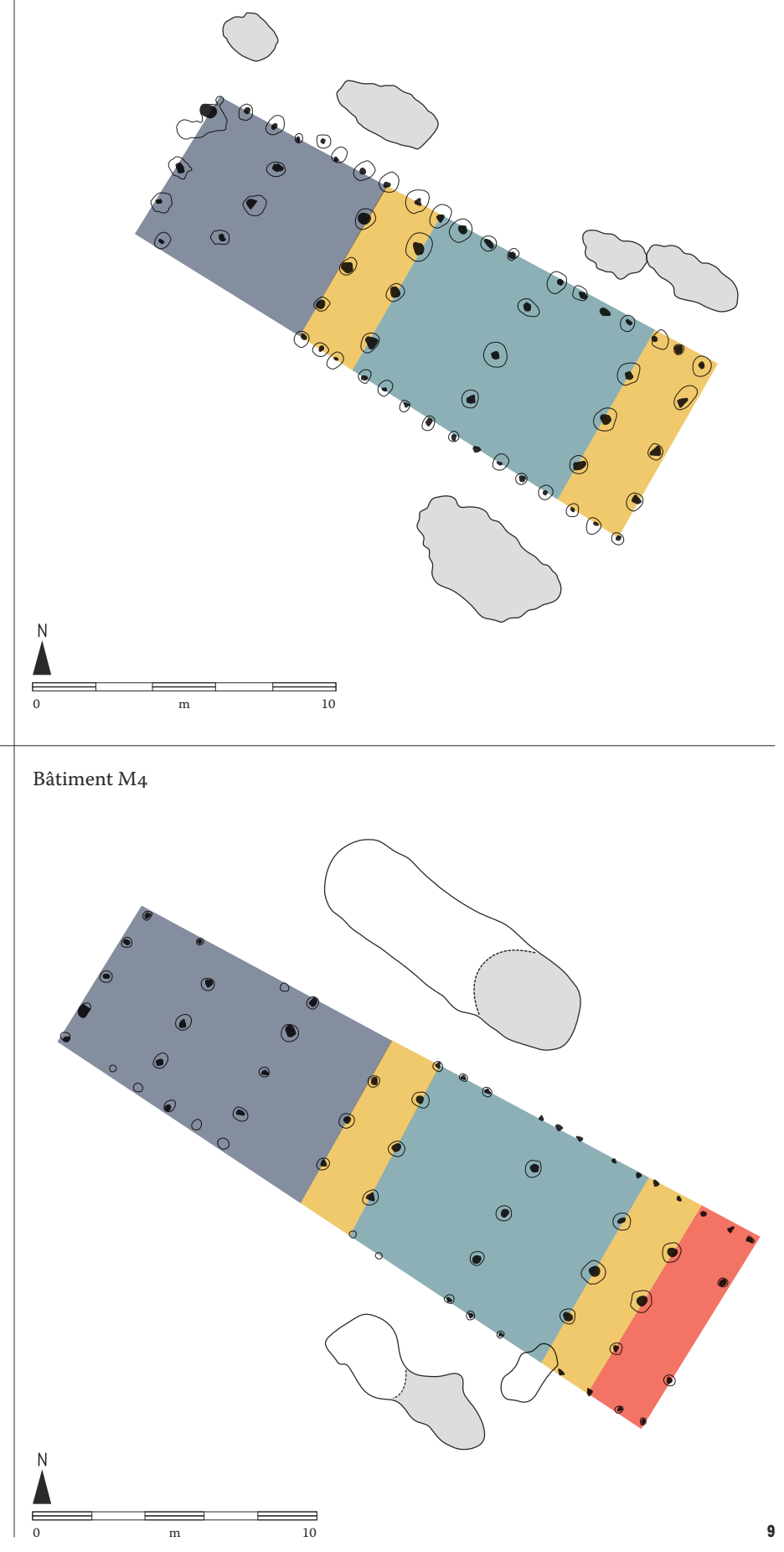

9. Évolution architecturale

$\begin{array}{ll}\square \text { partie frontale } & \square \text { partie arrière } \\ \square \text { partie centrale } & \square \text { corridor }\end{array}$ 
et des carporestes corrobore l'existence d'une aire de circulation et d'évacuation des déchets correspondant à l'entrée du bâtiment. Dans la partie centrale composée de quatre travées se dessine une tripartition de l'espace. La concentration des nodules de terre brûlée, dans les première et deuxième travées, indique vraisemblablement l'emplacement du four domestique. Dans les troisième et quatrième travées, les carporestes se concentrent dans la moitié sud, qui correspond ainsi possiblement au lieu des activités culinaires. Au niveau du corridor arrière, les densités importantes de carporestes ainsi que le spectre carpologique semblable à celui trouvé dans la fosse 1089 située en face, le long de la paroi nord, suggèrent l'existence d'une aire de circulation et d'évacuation des déchets du secteur nord du bâtiment. Dans la partie arrière, la quasi-absence de microvestiges mais les concentrations importantes de carporestes pourraient quant à elles résulter d'un espace consacré au stockage de denrées alimentaires.

Des occurrences similaires sont observables, avec quelques variantes, sur les autres bâtiments de Marainville, et la méthode permet de mettre en évidence une évolution chronologique intra-site [ill. 9]. Ainsi, le corridor avant, placé en façade dans la première phase d'occupation (M5 et M1) est, à partir du Rubané final (M2 et $\mathrm{M}_{4}$ ), précédé d'un auvent faiblement ancré dans le sol et qui protège le pignon. La partie centrale, composée de quatre travées dans la maison $M_{5}$, correspondant à la phase d'installation, se réduit à deux travées lors des occupations suivantes, ce qui pourrait traduire le passage d'une famille élargie à une famille nucléaire. Enfin, on note une augmentation de la surface occupée par la partie arrière, utilisée pour le stockage des denrées, qui passe de $30 \mathrm{~m}^{2}$ dans la maison $\mathrm{M}_{5}$ à $60 \mathrm{~m}^{2}$ dans la maison $\mathrm{M}_{4}$, qui est la plus récente du site.

La généralisation de ce type d'études et la constitution de séries significatives devraient permettre de mieux cerner l'évolution et les éventuels particularismes régionaux des habitats rubanés. Le développement de cette méthodologie nécessiterait la mise en place d'un protocole normalisé pour le prélèvement et le tamisage du remplissage du négatif des poteaux.

\section{Références bibliographiques}

BAKELS C. C., 1978, Four Linearbandkeramik settlements and their environment: a paleoecological study of Sittard, Stein, Elsloo and Hienheim, Leiden, Leiden University Press, « Analecta Praehistorica Leidensia » 11, 24.4 p.

Barclay G. J., Brophy K., MacGregor G., 2002, "Claish, Stirling: an early Neolithic structure in its context », Proceedings of the Society of Antiquaries of Scotland, 132, p. 65-137.

BERRIO L., 2012, Économie végétale et alimentation en Lorraine au Néolithique ancien. Étude carpologique du site rubané de Marainville-sur-Madon «sous le Chemin de Naviot » (Vosges). vol. 1 Texte, Mémoire de Master 2, Université Paris 1 Panthéon-Sorbonne, UFR Histoire de l'art et archéologie, Paris, $170 \mathrm{p}$.

Blouet V., 2006, « Du Néolithique au Bronze ancien entre Pont-à-Mousson et Thionville : Bilan sommaire de vingt-cinq ans d'archéologie préventive dans un terroir du bassin de la Moselle ", in BRUN P., Marcigny C., Vanmoerkerke J. (dir.), Une archéologie des réseaux locaux. Quelles surfaces étudier pour quelle représentativité ?, Les Nouvelles de l'Archéologie,104-105, Paris, Éditions Errance, p. 29-34.

BLOUET V., 2017, « Ni anges ni démons : de la violence chez les premiers agriculteurs de l'Europe du NordOuest ", in Manolakakis L., Schlanger N., Coudart A. (dir.), Archéologie européenne, Identité et migrations, hommages à Jean-Paul Demoule, Leiden, Sidestone Press, p. 339-370.

Blouet V., Decker E., Klag T., Petitdidier M.-P. Thomashausen L. et al., 2007, Le Néolithique ancien en Lorraine, Rapport d'Action Collective de Recherche, Metz, SRA de Lorraine, dactyl., 3 vol., $298 \mathrm{p}$.

Blouet V., Klag T., Petitdidier M.-P., Thomashausen L., 2013, Le Néolithique ancien en Lorraine, Paris, Société préhistorique française, «Mémoire » 55, vol. 1, 377 p. ; vol. 2, 36o p.

Bog AARD A., 2011, Plant use and crop husbandry in an early Neolithic village: Vaihingen an der Enz, BadenWürttemberg, Bonn, Verlag Dr. Rudolf Habelt GmbH «Frankfurter Archäologische Schriften » 16, 391 p.
Coudart A., 1998, Architecture et société néolithique: l'unité et la variance de la maison danubienne, Paris, Maison des sciences de l'homme, « Documents d'archéologie française » 67, $239 \mathrm{p}$.

De HingH A. E., 20oo, Food Production and food procurement in the Bronze Age and Early Iron Age (200o-50o BC): the organisation of a diversified and intensified agrarian system in the Meuse-DemerScheldt region (The Netherlands and Belgium) and the region of the river Moselle (Luxemburg and France), Archaeological Studies Leiden University 7, Leiden, Faculty of Archaeology, 235 p.

Fairweather A. D., Ralston I. B. M., 1993, « The Neolithic timber hall at Balbridie, Grampian Region, Scotland: the building, the date, the plant macrofossils ", Antiquity, 67, 255, p. 313-323.

Kirby M., 2011, « Lockerbie Academy: Neolithic and Early Historic timber halls, a Bronze Age cemetery, an undated enclosure and a post-medieval corndrying kiln in south-west Scotland ", Scottish Archaeological Internet Report, 46, 67 p.

KNöRZER K.-H., 1988, « Untersuchungen der Früchte und Samen », in Boelicke U., von Brandt D., LÜNING J., Stehli P., Zimmermann A. (dir.), Der bandkeramische Siedlungsplatz Langweiler 8: Gemeinde Aldenhoven, Kreis Düren, Köln, Rheinland-Verlag GmbH, « Beiträge zur neolithischen Besiedlung der Aldenhovener Platte III; Rheinische Ausgrabungen » 28, p. 813-852.

Kreuz A., 2012, « Die Vertreibung aus dem Paradies? Archäobiologische Ergebnisse zum Frühneolithikum im westlichen Mitteleuropa », Berichte de RömischGermanischen Kommission, 91, 2010, p. 23-196.

Kreuz A., Marinova E., Schäfer E., Wiethold J., 2005, «A comparison of early Neolithic crop and weed assemblages from the Linearbandkeramik and the Bulgarian Neolithic cultures: differences and similarities ", Vegetation History and Archaeobotany, 14,4 , p. 237-258.
Lenneis E., Trebsche P., 2013, « Die Dokumentation des nachgebauten linearbandkeramischen Langhauses von 1964. Ein Beitrag zur Diskussion der möglichen Lebensdauer ", in LAUERMANN E. (dir.), Das " jungsteinzeitliche » Langhaus in Asparn an der Zaya: Urgeschichte zwischen Befund und Experiment, Sankt Pölten, Niederösterreichisches Institut für Landeskunde, « Archäologische Forschungen in Niederösterreich » 11, p. 87-145

LÜNING J., 1998, « L'organisation régionale des habitats rubanés : sites centraux et sites secondaires (groupements de sites) », in CAUwE N., VAN Berg P.-L. (dir.), Organisation néolithique de l'espace en Europe du Nord-Ouest, Actes du XXIII ${ }^{e}$ Colloque interrégional sur le Néolithique, Bruxelles, Société royale belge, «Anthropologie et préhistoire » 109, p. $163-185$.

Murray H. K., Murray J. C., Fraser S. M., 2009, A Tale of the Unknown Unknowns: a Mesolithic pit alignment and a Neolithic timber hall at Warren Field, Crathes, Aberdeenshire, Oxford, Oxbow books, $132 \mathrm{p}$.

PAVLŮ I., 200o, Life on a Neolithic site Bylany: Situational Analysis of Artefacts - Život na sídlišti kulturys lineární keramikou v Bylanech u Kutné Hory: situační analýza artefaktů, Praha, Institute of Archaeology CAS, $340 \mathrm{p}$

Petitdidier M.-P., avec la collaboration de Arbogast R.-M., Blouet V., De Hingh A., Thomashausen L., Véber C., 2006, Ennery, Flévy, Trémery "ZAC de la Fontaine des Saints-Voirie » (Moselle), avril à septembre 1995, rapport d'opération, Inrap-SRA de Lorraine, $272 \mathrm{p}$.

PÉTREQUin P., 1984, Gens de l'Eau, Gens de la Terre. Ethnoarchéologie des communautés lacustres, Paris, Hachette, 345 p.

ReynOlds P. J., 1995, « Chapter 4: The Life and Death of a Post-Hole », in SHepherd E. (dir.), Interpreting Stratigraphy 5 , Proceedings of a Conference held at Norwich Castle Museum on 16th June 1994 and supported by the Norfolk Archaeological Unit Norwich, Norfolk Museum Services, p. 21-25

SouDSKÝ B., 1969, «Étude de la maison néolithique », Slovenská Archeológia, XVII, 1, p. 5-96. 